\title{
Knowledge and ability assessment of endodontists to prescribe and analyze cone beam computed tomography
}

\begin{abstract}
Objective: To determine the prevalence of knowledge about cone-beam computed tomography (CBCT) by endodontists from the Metropolitan Area of Belém, Pará, Brazil.

Material and methods: 151 questionnaires were spread containing 32 questions, 15 closed questions, 4 open and 13 mixed. Questions 1 to 17 dealt with questions such as age; gender; geographic location where endodontists' office operates; year of graduation degree and of specialization in endodontics; owned equipment and questions about the conventional radiology practice at dental office. Questions 18 to 32 were related to knowledge about the $\mathrm{CBCT}$ and only those who reported having knowledge answered the questions 19 onwards.

Results: 81 questionnaires were analyzed. 48 endodontists (59\%) had some knowledge about $\mathrm{CBCT}$, but among them, 5 never requested until that occasion. Regarding the radiation level, $76.75 \%$ have not any information about. The treatment plan of $36(83.72 \%)$ those who requested $\mathrm{CBCT}$ changed.
\end{abstract}

Conclusion: The CBCT imaging is slowly becoming an essential modality of imaging in the clinical practice of endodontists from the Metropolitan Area of Belém, Para, Brazil.
Volume 12 Issue 2 - 202 I

\author{
Monica Naomi Seko,' Pedro Luiz de \\ Carvalho,' Erick Alves dos Santos,' Larissa \\ Victória Barbosa Freitas,' Edson Marcos \\ Leal Soares Ramos, ${ }^{2}$ Afonso Celso Souza de \\ Assis, ${ }^{3}$ Kunihiro Saito,' João Marcelo Ferreira \\ de Medeiros ${ }^{4}$ \\ 'School of Dentistry, Federal University of Pará, Brazil \\ ${ }^{2}$ School of Statistical, Federal University of Pará, Brazil \\ ${ }^{3}$ School of Dentistry, University of Taubaté, Brazil \\ ${ }^{4}$ School of Dentistry, University Brazil, Brazil
}

Correspondence: Pedro Luiz de Carvalho, Rua Augusto Corrêa, 01 - Guamá, CEP 66075-I I0, Belém, Pará, Brazil, Email pedrolc@ufpa.br

Received: March 22, 202I | Published: March 29, 202 |

Keywords: endodontics, knowledge, surveys, tomography

\section{Introduction}

Radiographic examination is essential not only for diagnosis, plan, and execution of endodontic treatment, but also to pathology follow-up, it is needed images before, during and after completion of treatment. The more precise the information for the diagnosis, the better the choice of treatment and more predictable the results. Even with the use of refined techniques, the images acquired using conventional radiographs show information in two-dimensional distribution (height and width). Relevant and valuable information in the third dimension (depth) are limited ${ }^{1}$, which may cause misinterpretation. These dimensions are also subject to distortion, regardless of used radiographic technique, in other words, standardization in monitoring recovery or worsening of small evolutions is not accurate.

Due to the low cost and the extensive knowledge acquired, periapical radiographs are still the most used in endodontic and dental procedures in general, providing detailed information to the professional. To overcome the limitations of conventional intraoral radiographs, numerous methods to complement them have been proposed, including, magnetic resonance (MR), ultrasonography, computed tomography (CT) and Cone-Beam Computed Tomography (CBCT). ${ }^{1}$

The advent of CBCT in modern dentistry promoted optimization processes in endodontic purposes approaches, improving diagnosis and treatment planning of complex clinical cases from the provision of three-dimensional images or two-dimensional with minimal distortion of the whole area and/or volume of root canals the endodontist. The CBCT is the diagnostic imaging method that is most developed and popularized in dentistry nowadays, motivated and explained by the cost-benefit between performance and low cost, combined with a dose of lower radiation compared to conventional CT., ${ }^{2,3}$ The technology of the $\mathrm{CBCT}$ was not made for replace conventional radiography, but to serve as an adjunct in the acquisition of information necessary for the proper therapeutic interventions.

The CBCT exam can get reconstructions of all conventional dental radiographs added to the information provided by multiplanar and $3 \mathrm{D}$ reconstructions. The image can be sent to prototyping, obtaining a model of the scanned region in silicone material, which is an interesting usage in Endodontic-Periodontal surgery planning, presenting possibilities to eliminate overlaps, the magnificent resolution attributed to the great image contrast and possibility to rebuild them in the axial, coronal, sagittal and oblique, as well as get a three-dimensional view of the structure of interest. ${ }^{4}$

The first CBCT equipment in Belém was installed 2008. Currently, all appliances are installed in clinics specialized in dental radiology. The objective of this study was to determine, through a questionnaire, the knowledge prevalence and prescription of CBCT exams by endodontists from the Belém, Pará, Brazil, as well as gather information about this knowledge and applicability in specific cases, also investigate whether there was change in treatment plan after receiving the results.

\section{Material and methods}

This study was approved by the Research Ethics Committee of the Institute of Science Health of the Federal University of Pará ICS/UFPA, Brazil. Opinion Number: 1979982. Terms of consent were obtained for all participants research and anonymity was guaranteed. For the construction of the questionnaire, the results of previous similar surveys served as a model..$^{5-7}$ The pilot questionnaire was applied, and changes were made. A list of 225 endodontists containing names and business addresses registered with the Regional 
Dental Council of Pará was obtained upon request. Inclusion criteria were the facts that they were endodontists, and they exercise their activities in Belém, Pará, Brazil. After the exclusion of 67 individuals by geolocation, there was obtained sample of 158 subjects. The computational tool OpenEpi version 3, for 158 endodontists universe was used to calculate the sample and was obtained the number of 113 participants for the confidence interval of $95 \%$ and 81 to $80 \%$. 151 questionnaires and terms of consent forms were distributed from the day October 28, 2016 during the VI Meeting of Endodontics of Pará and after, in public and private offices by personal delivery and direct approach, preceded by telephone contact, discarding the post. The receiving happened until 23 March 2017. 97 filled questionnaires were obtained, however 16 individuals who work in other cities or did not deliver the consent were excluded. Therefore, 81 questionnaires were analyzed.

Each questionnaire contained 32 questions among which 15 closed, 13 open and 4 mixed, in other words, with multiple-choice questions, but with space for their opinion. It was answered by the participant without any interference, allowing it to express freely, and at least one day after delivery, collected by the researcher. At questions 1 to 17 were included questions about age, gender, demographic location where they execute their activities as well as employment bond, year of graduation and year of specialization in endodontics, equipment used in procedures and questions regarding the practice of conventional radiology in the office. Only issues 18 to 32 were concerning knowledge about the $\mathrm{CBCT}$ and only those who claim to have knowledge about answered the questions 19 onwards. These related to how often and in which situation where requested CBCT exam, if they had expected the outcome or not before starting treatment, if they knew the radiation dose, their preferable recording type, if they knew what the word "voxel" means, if they have done a course about CBCT, which upgrade methods were used and finally if the exam result changed the treatment plan.

Data analysis was performed by using the statistical technique, Descriptive Analysis using the Excel software, specifically with the aid of tables and graphics. In the statistical analysis for the variables "has knowledge of CBCT" and "Has another specialty" were applied Fisher's exact test in BioEstat software version 5.3 with a significance level of $95 \%(\alpha=0.05)$.

\section{Results}

Great part of the investigated individuals was composed by women (75\%). Considering the age distribution, the highest percentage $(32.5 \%)$ had between 41 to 50 years, followed by the portion between 31 to $40(27.5 \%)$, between 51 to 60 years $(21.25 \%)$, between 21 to 30 years $(11.25 \%)$ and the last parcel more than 61 years old $(7.5 \%)$.

Regarding time since graduation, approximately $50 \%$ of the participants had, in average, 19 years of experience; however, the most cited time (mode) was 31 years. Concerning the period as endodontist, the average was approximately 15 years and the amplitude was 37 years (Table 1).

Table I Variables Statistics:Age, Time since graduation and Time since specialization from research participants

\begin{tabular}{llllllll}
\hline \multirow{2}{*}{ Variable } & \multicolumn{2}{l}{ Statistics (years) } & \multicolumn{1}{l}{} \\
\cline { 2 - 8 } & Average & Median & Mode & $\begin{array}{l}\text { Standard } \\
\text { deviation }\end{array}$ & Amplitude & Minimum & Maximum \\
\hline Age & 43.32 & 44 & 53 & 10.89 & 58 & 24 & 82 \\
$\begin{array}{l}\text { Time since } \\
\text { graduation }\end{array}$ & 19.81 & 19 & 31 & 10.95 & 60 & 2 & 62 \\
$\begin{array}{l}\text { Time since } \\
\text { specialization }\end{array}$ & 14.75 & 13 & 11 & 9.21 & 37 & 0 & 37 \\
\hline
\end{tabular}

Source: research protocol

A Great parcel $(36.25 \%)$ works only at private dental office. Followed by those who works both in private dental office and in public institutions $(27-50 \%)$ (Table 2). Regarding the periapical radiographs requested by endodontists, there was a slight preference for the parallelism technique over the bisector. Concerning the method of processing radiographic films, only two reported the use of digital radiography. In addition, $79.74 \%$ request imaging tests from the specialist in clinical radiology. As for possessed equipment, among others commonly found, 4 endodontists have surgical microscopes.

Table 2 Amount and percentage of research participants by type of establishment where execute their activities

\begin{tabular}{lll}
\hline Type of establishment where execute their activities & Amount & Percentage \\
\hline Private Dental Office & 29 & 36.25 \\
Private Dental Office and Public Institution & 22 & 27.50 \\
Public Institution & 12 & 15.00 \\
Private Clinic & 5 & 6.25 \\
Public Institution and Others & 3 & 3.75 \\
Private Dental Office and Private Clinic & 3 & 3.75 \\
Private Dental Office, Private Clinic and Public Institution & 3 & 3.75 \\
University Town / City - University Center & 1 & 1.25 \\
Private Dental Office and Others & 1 & 1.25 \\
Private Dental Office, Public Institution and Others & $\mathrm{I}$ & 1.25 \\
& & 100.00 \\
Total & 80 & \\
\hline
\end{tabular}

Source: research protocol

Note: I participant has not provided any information

Citation: Seko MN, Carvalho PL, Santos EA, et al. Knowledge and ability assessment of endodontists to prescribe and analyze cone beam computed tomography.J Dent Health Oral Disord Ther. 202I;I2(2):27-3I. DOI: 10.15406/jdhodt.202I.12.00545 
As for knowledge about the technology, 48 interviewees (59\%) had some knowledge about CBCT; however, among them, 5 have never requested so far. Of these, 3 related the high monetary cost as a reason, 1 replied that it was not necessary to request and 1 answered that had no knowledge to do so. In addition, 35 interviewees (43.21\%) report having other additional specialties in Endodontics, such as Implantology, Orthodontics, Prosthesis, and others (Table 3).

Table 3 Demonstration of the relationship between CBCT knowledge and having another specialty

\begin{tabular}{lllll}
\hline \multirow{2}{*}{ Have knowledge of CBCT } & \multicolumn{2}{l}{ Does have other specialty } & Total & P-Value \\
\cline { 2 - 4 } & No & Yes & \\
\hline Have knowledge & 10 & 25 & 46 & 0.0687 \\
Do not have knowledge & 23 & 23 & 35 & \\
Total & 33 & 48 & 81 & \\
\hline
\end{tabular}

Source: research protocol

As for the request frequency, of 43 interviewees that already requested this type of radiographic examination, $2.38 \%$ request it up to 4 times a week, $23.81 \%$ about once a month, $26.19 \%$ once a week and $47.62 \%$ rarely request. There was no selection option more than 5 times per week. Table 4 shows that the most common situation for requesting a $\mathrm{CBCT}$ scan was suspicious of root fracture and perforation, followed by visualization of resorption areas. Finally, there is the morphology of the root canal system and to detect the exact location of the fractured instruments. In this question, the principle of multiple choices was used.

Table 4 Amount and percentage of research participants per case of $C B C T$ prescription, per preferred recording format, and per Means to update the knowledge about tomographic image

\begin{tabular}{lll}
\hline Variable & Amount & Percentage (\%) \\
\hline CBCT Prescription Case* & & \\
Suspicious of fracture & 40 & 29.1 \\
Suspicious of perforation & 38 & 27.2 \\
Visualization of resorption areas & 25 & 18.2 \\
Visualization of the morphology of root canal system & 16 & 11.6 \\
To detect the exact location of fractured instruments & 15 & 10.9 \\
Others & 3 & 2.1 \\
& & \\
Preferred Recording Format & & \\
JPEG (a) & 5 & 12.5 \\
DICOM (b) & 6 & 15.0 \\
Both (a) and (b) & 12 & 30.0 \\
Analyze only printed images and radiologist report & 17 & 42.5 \\
Used means to update the knowledge about tomographic image*** & & \\
& & \\
Conferences & 13 & 28.88 \\
Conferences and Internet & 6 & 13.34 \\
Internet & 5 & 11.12 \\
Conferences and Courses & 4 & 8.89 \\
Conferences, Books and Journals & 3 & 6.67 \\
Courses, Book and Journals & 3 & 6.67 \\
Courses & 2 & 4.44 \\
Internet, Books and Journals & 2 & 4.44 \\
Books and Journals & 2 & 4.44 \\
Conferences, Internet, Books and Journals & 2 & 4.44 \\
Conferences, Internet, Courses, Books and Journals & 2 & 4.44 \\
Courses and Internet & 1 & 2.23 \\
\hline
\end{tabular}

Source: research protocol

Note: $* 2$ participants have not provided any information

Note: $* * 3$ participants have not provided any information

To the question: "Starts treatment before receiving CBCT results?", $45 \%$ said no, $5 \%$ said they started and 50\% said, depending on the case, yes. In the place reserved for the case description of the latter alternative, most reported that it was due to pain.
As to the radiation level, 33 participants did not know to inform and only 10 participants declared to have knowledge. Among them, 3 answered it is like full-mouth periapical radiography, other 5 that depends on the FOV (Field of view). One participant declared that it 
is like panoramic radiography other pointed out that $\mathrm{CBCT}$ is like any other computed tomography.

Regarding the preferred recording format, $42.5 \%$ chose the "only printed images and radiologist report" option and only $15 \%$ preferred the DICOM format (Table V). However, in the next question, 18 participants $(41.86 \%)$ admitted that they know to manipulate the images on computer.

About $93 \%$ of participants usually show and explain tomographic imaging to patients. But just over half $(57.77 \%)$ know the meaning of "voxel size". The most used means to update the knowledge of tomographic images are conferences $(28.88 \%)$, association conferences and internet $(13.34 \%)$, and can choose more than one answer (Table 4). Only 12 said that had attended CBCT courses.

Finally, concerning the question if the result of the CBCT modified treatment plan or not, $36(83.72 \%)$ answered yes, citing the root fracture and the extraction as the main reason of it. Perforation and visualization of resorption areas have been mentioned, as well as show calcification of root canals.

\section{Discussion}

The response rate was 81 completed questionnaires for 151 delivered (53.64\%), is lower when compared to European countries such as Sweden and Norway. ${ }^{5,6}$ The biggest difficulties were outdated information; number of retired professionals; refusal to participate in the research; and many professionals complete the specialization in Endodontics, but do not register the specialty. Experts in general have extensive experience in diagnostics using traditional imaging modes (intraoral radiography and maxillary-mandibular panoramic extraoral imaging), where the confidence level in interpreting these images is high. ${ }^{4}$ The radiographic procedures of pathology followup obtained using the periapical technique by parallelism principle is not consistently reproducible, which may result in underestimation or overestimation of the actual cure or failure of endodontic treatment. The biggest challenge for using CBCT is the lack of familiarity with the concept of three-dimensional imaging. The overlap and interference of anatomy, added to geometric distortion and are part of everyday life, but also considering CBCT as a new technology, many endodontists have not requested, perhaps due to lack of knowledge of the indications and limitations of the test. Knowledge about CBCT is gradually inserted in Brazilian universities and specialized courses. Moreover, although the technology is complex, the result submitted is easy to understand and undoubtedly a valuable complementary test to add to the endodontist's arsenal.

Regarding the cost, CBCT technology is evolving at a rapid pace. At the same time more companies are introducing bigger and more sensitive CBCT sensors in a competitive market and steady expansion. This may result in a reduction in these sensors cost, which in turn will increase their adoption by dentists 9 . In the other hand, operators of CBCT equipment should be adequately trained, as well as for interpreting the images because $\mathrm{CBCT}$ is completely different from conventional scheme. ${ }^{9}$

The CBCT image has four production components: acquisition setup, image detection, image reconstruction, and image display. The image is obtained by the ability to rotate the x-ray gun and an image receiver at positions $180-360^{\circ}$ adjacent to the patient. Time reconstruction varies depending on the acquisition parameters (voxel size and number of FOV projections) of hardware (processing speed, acquisition data flow to the computer workstation) and software (reconstruction algorithms) used. Reconstruction should be performed in less than 3 minutes for standard resolution examinations to monitor patient flow. ${ }^{10}$

However, assuming that there are different needs for each specialty, the endodontist should know the limitations when it comes to artifacts. Depending on how the tooth is sent to the examination, the reason for failure in reconstruction, teeth with metal pins, gutta percha points and calcium hydroxide itself generate some difficulty in the view. Fractured instruments surrounded by gutta percha are not identifiable. So, as far as possible, to increase the efficiency of the examination, convenient would be the withdrawal of any material that interferes with image production, because depending on the clinical case, further radiographs are superior.

Radiation should be known to justify the safety of this method for the most concerned patients. The unfamiliarity of the radiation level by most of the respondents may influence when requesting the area to be studied, as lower FOVs are related to lower radiation levels. The endodontist is not expected to know the technology as a radiologist, but the endodontics is the specialty that requires smaller voxel sizes to increase accuracy. The kilovoltage, and sometimes the milliampere, as well as the exposure time are increased as indicated and the area to be studied. The ALARA (As Low as Reasonably Achievable) principle within radiological protection establishes in its basic philosophy the reduction of exposure to the lowest possible dose levels, dose ratios and environments propagation, and its scope includes a wide range of factors to consider, such as: technical, administrative, economic and social.

Compared to conventional radiographs, the $\mathrm{CBCT}$ radiation dose is like that of the full-mouth periapical examination or is approximately 4 to 15 times the dose of a maxillary-mandibular panoramic radiograph and is being widely used in the dental area. ${ }^{10}$ One of the biggest advantages of $\mathrm{CBCT}$ over $\mathrm{CT}$ is the effective dose, significantly lower to which patients are exposed. The effective dose of $\mathrm{CBCT}$ sensors varies but can be almost as low as maxillary mandibular panoramic radiography and considerably smaller than $\mathrm{CT}$.

In endodontic practice, $\mathrm{CBCT}$ is useful both in the differential diagnosis between pathologies of endodontic and non-endodontic origin, and as a fundamental complementary exam for good clinical practices and treatment success, such as: evaluation of alveolar and root fractures; evaluation of root canal morphology (main canal, collateral canal, lateral canal, secondary canal, accessory canal, interconduct, recurrent canal, reticular channels and apical delta); localization of pulp calcifications; internal and external root resorption analysis; preoperative endodontic planning; evaluation of root preparation; obturation; retreatment; bone lesion detection and endodontic research. ${ }^{8}$ As for morphology visualization, CBCT scans are invaluable for analyzing teeth with unusual anatomy, such as teeth with atypical root numbers, root lacerations and dens invaginatus. The exact location and root canal system can be analyzed, allowing for successful case management.

The periapical pathologies can be detected early with the use of $\mathrm{CBCT}$ compared to periapical radiographs, showing the true size, nature, and position of osteolytic lesions. ${ }^{8,11,12}$ Nevertheless, in Estrela's study ${ }^{13}$, inflammatory root resorption associated with canal infection and periapical lesions were found in $61.4 \%$ of the cases studied by scanning electron microscopy and at least half of the cases studied by CBCT. In microscopic analysis remains the reference pattern in contrast to other methods to identify this resorption. It can be speculated that CBCT may be improved over time and in the future will improve the diagnosis for this clinical situation. 
As the product of the exam approached are hundreds of images accompanied by a technical report, it is essential to know the manipulation of these images on the computer; otherwise, the diagnosis will always depend only on the radiologist's knowledge and expertise. At Belém, Pará, Brazil, the equipment is in dental radiology clinics, where they provide results printed on film, paper, electronic media, or electronic mail. Therefore, it is necessary to have a reader installed on the computer and knowledge of the DICOM format. Moreover, in countries where the technology is commonly used, the $\mathrm{CBCT}$ is installed in non-specialized clinics and knowledge has become routine..$^{5,6}$

The CBCT imaging is slowly becoming an essential modality of imaging in the clinical practice of Belém, Pará, Brazil professionals. For effective use of this technology, it is necessary to know not only the advantages but also the limitations. Although new high-definition software technologies and artifact reduction have eliminated these limitations to negligible levels, every clinician should be able to evaluate and manipulate tomographic images.

\section{Acknowledgments}

None.

\section{Conflicts of interest}

None.

\section{References}

1. Weber MT, Stratz N, Fleiner J, et al. Possibilities and limits of imaging endodontic structures with cbct. Swiss Dent J. 2015;125:293-311.

2. Leonardi Dutra K, Haas L, Porporatti AL, et al. Diagnostic accuracy of cone-beam computed tomography and conventional radiography on apical periodontitis: a systematic review and meta-analysis. $J$ Endod. 2016;42(3):356-364.
3. AAE and AAOMR joint position statement. Use of cone beam computed tomography in endodontics 2015 update. J Endod. 2015;120(41):13931396.

4. Mota de Almeida FJ, Knutsson K, Flygare L. The impact of cone beam computed tomography on the choice of endodontic diagnosis. Int Endod J. 2015;48(6):564-572.

5. Hol C, Hellén-Halme K, Torgersen G, et al. How do dentists use CBCT in dental clinics? A Norwegian nationwide survey. Acta Odontol Scand. 2015;73(3):195-201.

6. Strindberg JE, Hol C, Torgersen G, et al. Comparison of Swedish and Norwegian use of cone-beam computed tomography: a questionnaire study. J Oral Maxillofac Res. 2015;6(4):e2.

7. Dölekoğlu S, Fişekçioğlu E, İlgüy $M$, et al. The usage of digital radiography and cone beam computed tomography among Turkish dentists. Dentomaxillofac Radiol. 2011;40(6):379-384.

8. Estrela C, Bueno MR, Azevedo B, et al. A new periapical index based on cone beam computed tomography. J Endod. 2008;34(11):1325-1331.

9. Venskutonis T, Plotino G, Juodzbalys G, et al. The importance of conebeam computed tomography in the management of endodontic problems: a review of the literature. J Endod. 2014;40(12):1895-1801.

10. Uraba S, Ebihara A, Komatsu K, et al. Ability of cone-beam computed tomography to detect periapical lesions that were not detected by periapical radiography: a retrospective assessment according to tooth group. J Endod. 2016;42(8):1186-1190.

11. Rodriguez G, Abella F, Duran-Sindreu F, et al. Influence of cone-beam computed tomography in clinical decision making among specialists. $J$ Endod. 2017;43(2):194-199.

12. Bueno Mike R. Development of a New Cone-Beam Computed Tomography Software for Endodontic Diagnosis. Braz Dent J. 2018;29(6):517-529.

13. Estrela C, Guedes OA, Rabelo LE, et al. Detection of apical inflammatory root resorption associated with periapical lesion using different methods. Braz Dent J. 2014;25(5):404-408. 